\section{Instabilities and Chaos in Lasers}

\author{
R.G. Harrison and J.S. Uppal,; Edinburgh
}

(Physics Department, Heriot-Watt University)

\begin{abstract}
Chaos and related dynamical instabilities are inherent features of many non-linear systems. The transition from a steady to chaotic state are universal, occurring independently of the physical properties of the system. These features occur abundantly in lasers which provide ideal systems for quantitative investigation of deterministic non-linear dynamics.
\end{abstract}

Since its advent more than a quarter of a century ago, the laser has evolved as a unique device for both fundamental investigation and applications in optical science and technology. In providing a coherent and intrinsically stable emission, lasers are fundamentally different from conventional light sources where the excited atoms (or molecules) emit spontaneously resulting in a signal comprising a statistical average of random or uncorrelated emissions. However, the conventional concept of laser emission as an ordered and time invariant process provides a description of but one aspect of operation. Motivated by profound mathematical discoveries in recent years which have revolutionised our understanding of non-linear science, it has been found that lasers, along with many other non-linear systems in a wide range of sciences, display a rich variety of dynamical characteristics which are common features of their operation [1].

As well as exhibiting regular and repeatable behaviour, these systems have also unstable, even chaotic solutions. Furthermore, the transition from stable to chaotic behaviour, on varying a control parameter of the system, e.g. in a laser: cavity tuning, excitation, etc., follows specific, well defined routes of temporal or dynamical behaviour which are universal in the sense that they are independent of the physical properties of the system they describe. It is these signatures which have given a major impetus to experimentalists in the subsequent search for physical systems that exhibit these phenomena. The exciting discovery of deterministic instabilities in lasers is particularly significant since they provide nearly ideal systems for quantitative investigations because of their simplicity both in construction and in the mathematics that describe them.

* J.S. Uppal is on leave from Laser Division, B.A.R.C. Bombay (India). (a)

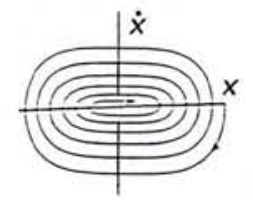

(b)
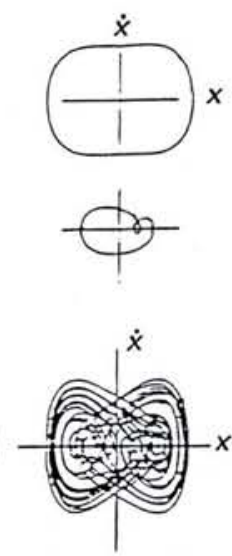

case different initial conditions lead to different types of long-time behaviour. The simplest attractor in phase space is a fixed point which is a solution with just one set of values for its dynamical variables; the non-linear system is attracted towards this point and stays there giving a DC solution. For other control conditions the system may end up making a periodic motion. The limit or attractor of this motion is a periodic cycle called a limit cycle. However, when the operating conditions exceed a certain critical value, the periodic motion of the system breaks down into a more complex chaotic pattern which never repeats itself. This motion represents a third kind of attractor in phase space called a chaotic or strange attractor.

The inherent feature of volume contraction in phase space of dissipative systems has a strong tendency to simplify the topological structure of the trajectories in phase space. This can often mean that a complex dynamical system with even an infinite-dimensional phase space - governed for example by partial differential equations - can settle to final behaviour in a subspace of only a few dimensions. To recognise such behaviour it is then essential that we first understand the dynamical behaviour through the topology of intrinsically lowdimensional mathematical models. The Duffing oscillator is one such example which describes the motion of a damped, periodically forced non-linear oscillator with displacement $(x)$ given by

$$
\ddot{x}+k \dot{x}+x^{3}=\beta \cos \omega t
$$

where $k$ is the damping coefficient and $\beta$ the amplitude of the oscillatory driving signal.
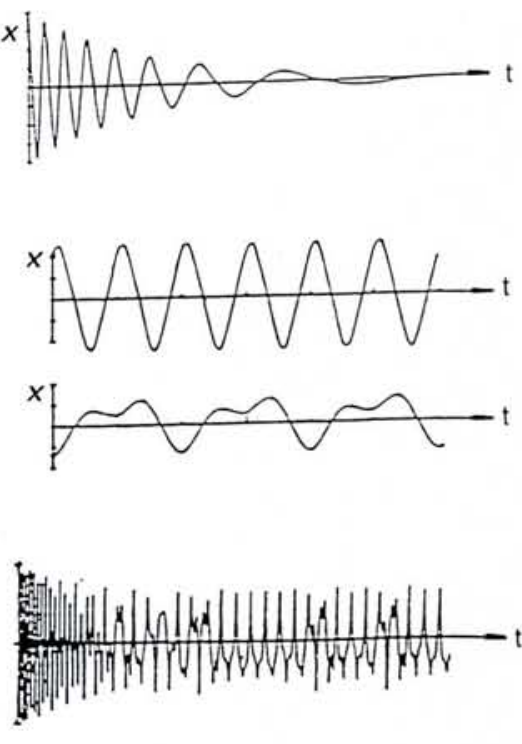

Fig. 1 - Behaviour of a Duffing oscillator; a) unforced behaviour showing damped transient oscillation to a fixed point; b) coexisting periodic or limit cycle attractors with different start conditions; c) transients setting to steady state chaos from ref. 2. 
Its behaviour can be described by the motion of a point in a three dimensional phase space whose coordinates are position, velocity and time of the oscillator's motion. Some representative solutions are shown in Fig. 1 [2]. A fixed point solution is obtained when the forcing term is set to zero, the system then relaxing to a unique stable equilibrium point (Fig. 1(a)) e.g. the rest position of a pendulum. Stable periodic motions are sustained by the presence of the driving term over a range of values of $k$ and $\beta$, the limit cycle behaviour also being determined by the initial, or start conditions (Fig. 1(b)). For other parameter values, the range of which is quite extensive, the behaviour breaks down into chaos. In a true three-dimensional representation, rather than the two-dimensional plot of Fig. 1(c), the trajectory, in contrast to those for a fixed point and limit cycle, never intersects itself, consistent with aperiodic behaviour. Evidently a system with three variables, or degrees of freedom provides the minimum number to ensure non-intersection of the trajectory in phase space.

By way of illustration, consider a ball of string where the string, representing the trajectory, intertwines and loops without intersecting (repeating itself) to form a finite volume (a strange attractor) in a three-dimensional phase space. For such parameter settings, arbitrarily close initial conditions lead to trajectories which after a sufficiently long time diverge widely. Since a truly exact assignment of the initial condition is never possible, even numerically, a solution comprising several such trajectories must therefore evolve and as a consequence long-term predictability is impossible. This is in marked contrast to that of the fixed point and limit cycle attractors which settle down to the same solutions.

Erratic and aperiodic temporal behaviour of any of the system's variables implies a corresponding continuous spectrum for its Fourier transform which is thus a further signature of chaotic motion. Time series, power spectra and routes to chaos collectively provide evidence of deterministic behaviour.

Of the wide range of possible routes to chaos, three have emerged as particularly common. These are period doubling [3], intermittency and two-frequency scenarios. In the first, a solution which is initially stable is found to oscillate, the period of which successively doubles at distinct values of the control parameter. This continues until the number of fixed points becomes infinite at a finite parameter value, where the varia-
Helsinki University of Technology

\section{Postdoctoral Fellowships}

and

\section{Sabbathical Leave Opportunities}

available in ultralow temperature physics (superfluid helium-3 in rotation) and brain research (magnetoencephalography).

Experimentalists and theorists welcome.

Six months - two years appointments. Competitive remuneration.

Write to Prof. Olli V. Lounasmaa, Low Temperature Laboratory,

TKK, 02150 Espoo, Finland.

\section{The Netherlands' \\ Foundation for Fundamental Research on Matter}

promotes fundamental scientific research on matter in the Netherlands generally and at universities in particular. The Foundation tries to achieve this purpose by stimulating research in new fields of physics, by co-ordinating existing research projects and by involving its institutes and working groups in the education and training of young physicists.

The Foundation has an opening for an

\section{Experimental Nuclear Physicist $(\mathbf{m} / \mathbf{f})$}

The Experimental Nuclear Physics group (EKF) of the Department of Physics and Astronomy of the Free University of Amsterdam invites applications for a tenured senior staff position for an experimental Nuclear Physicist in a "senior onderzoeker" rank. For a highly qualified and experienced applicant an appointment in a "hoofd onderzoeker" rank may be possible. In the Dutch system, the "senior and hoofd onderzoeker" ranks are comparable, respectively, to assistant and associate professor ranks in the American system. The appointment will be with the Netherlands Foundation for Fundamental Research on Matter (FOM).

The EKF concentrates its research programmes at the two Dutch National Institutes for Nuclear Research: the NIKHEF-K in Amsterdam and the KVI in Groningen. The NIKHEF-K facility has a medium energy electron accelerator with a maximum energy of $600 \mathrm{MeV}$. Recently the NIKHEF-K project for updating this accelerator to increase the energy to $700 \mathrm{MeV}$ and to build a stretcher ring thereby increasing the duty factor to $90 \%$ has been approved. The $\mathrm{KVI}$ has at the moment an AVF-cyclotron $(K=160 \mathrm{MeV})$ for the acceleration of light and heavy ions. At present the $\mathrm{KVI}$ is building in collaboration with the Institut de Physique Nucléaire in Orsay a superconducting cyclotron for heavy and light ions, AGOR $(K=600 \mathrm{MeV})$, to replace the present cyclotron in 1991.

Applicants should be interested in the research of the EKF which is performed at both facilities with special emphasis on the research programme carried on at the KVI. Applicants should be capable of initiating new lines of research, should take responsibilities for developing new instruments especially for NIKHEF-K, and should have good leadership qualities and teaching skills.

Information about the position can be obtained from prof.dr. M.N. Harakeh, tel. (20) 5487521 . Those who may want to draw our attention to qualified candidates are kindly requested to contact prof.dr. M.N. Harakeh.

Applications with curriculum vitae and the names of three references should be sent within three weeks from the appearance of this advertisement to

dr. J.J. Vasmel, Vrije Universiteit,

Postbus 7161, NL-1007 MC Amsterdam, the Netherlands. 
tion in time of the solutions becomes irregular. For the intermittency route, a signal that behaves regularly in time becomes interrupted by statistically distributed periods of irregular motion, the average number of which increases with the external control parameter until the condition becomes totally chaotic. The two frequency route is more readily identified with early concepts of turbulence, considered to be the limit of an infinite sequence of instabilities (Hopf bifurcation) evolving from an initial stable solution each of which creates a new basic frequency. It is now known that cnly two or perhaps three instabilities (frequencies) are sufficient for the subsequent generation of chaotic motion.

\section{Chaos in Lasers}

Our conventional understanding of laser physics is concerned with how cooperative order evolves from randomness. This transition is explained by first considering the ensemble of lasing atoms in thermal equilibrium. Every atom executes a small motion, giving rise to an oscillating electric dipole, described by linear dynamics. For larger motions, the atomic dipoles have to interfere with one another's motion and beyond a particular threshold, the motion becomes "cooperative" or ordered over long range. The role of the e.m. field in the laser cavity in ordering the induced atomic dipoles is collectively described as giving rise to a macroscopic polarisation, the magnitude of which depends on the number of dipoles (excited atoms). The dynamical interplay between the cavity field as one variable and the atomic material variables of polarisation and population of excited atoms, all of which are in general complex quantities, provide a full description of lasing action. For the simplest laser, a single mode 2-level system with a homogeneously broadened gain, these reduce to just three real variables.

Described in this way the laser acts as a single oscillator in much the same way as the mechanical non-linear oscillator considered earlier, where laser output represents damping of the oscillator and excitation of the atoms is the driving mechanism to sustain lasing (oscillation). For ordered or coherent emission, it is however necessary for one or both of the material variables (which are responsible for further lasing emission) to respond sufficiently fast to ensure a phase correlation with the existing cavity field. This is readily obtained in many typical lasers with output mirrors of relatively high reflectivity, since variations in field amplitude within the laser cavity will then vary slowly compared to

\section{Maxwell-Bloch}

$\dot{E}=-\kappa E+\kappa P$

$\dot{P}=\gamma_{\perp} E D-\gamma_{\perp} P$

$\dot{D}=\gamma_{\|}(\lambda+1)-\gamma_{1} D-\gamma_{\|} \lambda E P$

\section{Lorenz}

$\dot{x}=-\sigma x+\sigma y$

$\dot{y}=-x z+r x-y$

$\dot{z}=x y-b z$

where for the Maxwell-Bloch equations $\kappa$ is the cavity decay rate, $\gamma_{\perp}$ is the decay rate of atomic polarisation, $\gamma_{\|}$is the decay rate of population inversion, $\lambda$ is the pumping parameter, $E$ is the field inside the cavity, $D$ is the population inversion and $P$ is the atomic polarisation. Consider the trajectory of the Lorenz attractor where in the equivalent laser system the dynamic variables $x, y$ and $z$ are the field amplitude $(E)$, polarisation of the medium $(P)$, and the population inversion (D).

the fast material variables which may then be considered through their equilibrium values. This situation, commonly referred to as adiabatic elimination of the fast variables, reduces the dynamics of lasing action to that of one (or two) variable, the field (and population), all others being slaved to adapt constantly to the slowly varying field variable.

Our familiar understanding of DC laser action presupposes such conditions to hold. However when for example the level of excitation of the atoms is increased to beyond a certain value, i.e. the second laser threshold, all the dynamical variables may have to be considered.
So even the simple single mode laser comprising three variables has a sufficient, though minimum, number of variables (or degrees of freedom) for the generation of chaos. Conceptually, then even this simple system is capable of chaotic motion, the lasing emission from which will be aperiodic in time and have a spectral content which is broad band. Prediction of such a behaviour was initially identified by Haken through observing the mathematical equivalence of Maxwell-Bloch equations describing the laser action [6] to those derived earlier describing chaotic motion in fluids [7] (see Table). (a)

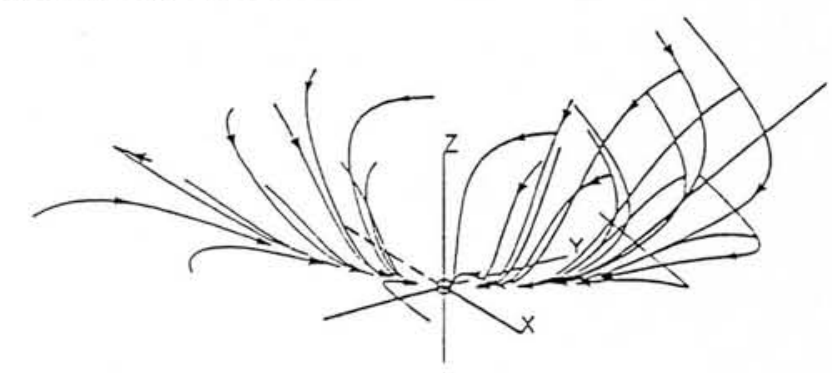

(b)
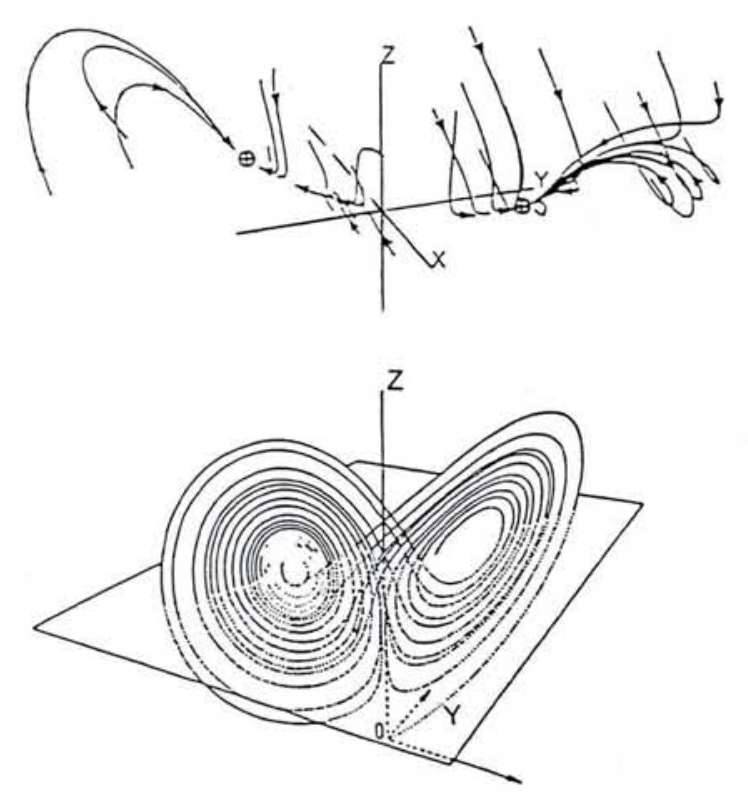

Fig. 2 - Trajectories in the three dimensional phase space of the Lorenz attractor.

a) Parameter $r$ is less than 1, and all trajectories approach stable equilibrium at the origin.

b) Trajectories attracted to two new stable equilibria for $r$ just greater than 1.

c) Chaotic trajectory for $r=28$. a) and b) from ref. 2 and c) from ref. 8. 
Fig. $2(a, b, c)$ shows a sequence of trajectories on increasing the value of one of the control parameters in the Lorenz equations. For the equivalent laser system, the dynamic variables $x, y$ and $z$ are the laser field amplitude, polarization of the lasing medium and population inversion of the medium respectively. For a control setting near zero, all trajectories approach stable equilibrium at the origin, the topological structure of the basin of attraction being hyperbolic about zero. For the laser equations, this corresponds to operation below lasing threshold for which the magnitude of the control parameter (laser gain) is insufficient to produce lasing. This solution is similar to the fixed point solution of the Duffing oscillator (see Fig. 1(a)) although the trajectory to the fixed point (transient behaviour) does not spiral in as for the latter.

As the control value is increased, the basin lifts at its zero point to create an unstable fixed point here but with now two additional fixed points located in the newly formed troughs on either side of the zero point which is now a saddle point. This is illustrated in Fig. 2(b) where the system eventually settles to one or other of the two new and symmetric fixed points depending on the initial conditions. This corresponds to DC or constant lasing as defined by the parameter values of one or other of these points which are indistinguishable. Pictorially think of a conventional saddle shape comprising a hyperbolic and inverted hyperbolic form in mutually perpendicular planes and connected tangentially at the origin. With the curve of the inverted hyperbola turned up at its extremity and filling in the volume with similar profiles which allow the two hyperbolic curves to merge into a topological volume, one sees that a ball placed at the origin is constrained to move most readily down either side of the inverted hyperbolas into one or other of the troughs formed by this volume. This topology may be loosely contrasted with that underlying limit cycle behaviour for the Duffing oscillator for which the original hyperbolic basin lifts at its zero point to create an annular trough about zero in which a ball will continuously orbit giving a periodic solution.

For the Lorenz system, chaotic behaviour occurs at larger values of the control parameter when all three fixed points become saddles. Since none of the equilibrium points is now attracting, the behaviour of the system cannot be a steady motion. Although perhaps difficult to visualise topologically, it is then possible to find a region in this surface enclosing all three points and large enough so that no trajectories leave the region. Thus all initial conditions outside the region evolve into the region and remain inside from then on. A corresponding chaotic trajectory is show in Fig. 2(c). A point outwardly spirals from the proximity of one of the new saddle points until the motion brings it under the influence of the symmetrically placed saddle, the trajectory then being towards the centre of this region from where outward spiralling again occurs. The spiralling out and switching over continues forever though the trajectory never intersects. A recording of one of these variables in time, say for a laser the output signal amplitude (in practice recorded as signal intensity, proportional to the square of the field amplitude), then gives oscillatory emission of increasing intensity with sudden discontinuities (resulting from the flipping from one saddle to the other of Fig. 2(c)) as expected. An experimental example of such behaviour for a single mode optically pumped far infrared molecular laser is shown in Fig. 3.

While the Lorenz/Haken model is attractive for its relative simplicity, many practical lasers cannot be reduced to this description. Nevertheless the underlying topology of the trajectory of solu-

\section{THE UNIVERSITY OF GRONINGEN THE NETHERLANDS ANNOUNCES AN OPENING FOR A}

\section{full professor of experimental physics $(\mathrm{f} / \mathrm{m})$}

(vacancy number: 880517/2493)

The appointee is expected to take a leading role in the research of the group Nuclear Solid State Physics. This group is part of the Groningen Materials Science Center (M.S.C.) which offers a broad spectrum of research projects in the field of condensed matter physics and chemistry.

The candidate is expected to take initiatives in the field of

\section{THE STRUCTURE OF INTERFACES AND THIN FILMS}

Present research in the Nuclear Solid State Physics group focuses on the electronic structure and lattice location of impurity atoms implanted in thin layers or deposited at interfaces. Another full professor in the group is specialized in the application of hyperfine interaction techniques to this research area.

The candidate will have at his/her disposal extended facilities for ion beam analysis from $5 \mathrm{keV}$ up to $5 \mathrm{MeV}$, in UHV conditions and at temperatures down to $25 \mathrm{~K}$, and a versatile high resolution isotope separator/ion implanter. Because the study of interfaces and thin films is a principal theme in the Materials Science Center, a large number of other facilities are available for their preparation and characterization.

Furthermore, the successful candidate is expected

- to conduct a high-quality research programme which makes it possible to obtain financial support from public funds

- to participate in the teaching programme of physic and applied physics, both at the undergraduate level and in the form of special lectures

- to supervise and inspire the research of undergraduate and Ph.D. students

- to participate in policy-making and administrative activities

- to acquire an adequate knowledge of the Dutch language within two years of the appointment.

Salary depending on experience to a maximum of Dfl 9621.- per month.

Letters of application, including a curriculum vitae, a list of publications and the names and addresses of two referees should be sent within four weeks after publication of this advertisement to the Director of Personnel, University of Groningen, Postbox 72, 9700 AB Groningen, The Netherlands, quoting our reference number 880517 .

Further information can be obtained from the chairman of the appointment committee, Prof. L. Niesen (phone 031-50-634736) or from the chairman of the Physics Department, Prof. N.M. Hugenholtz (phone 031-50-634962).

Those wishing to recommend potential candidates are invited to send a letter to the Director of Personnel.

In line with our policy we invite especially women to apply for our advertised posts. 
tions in phase space for these systems is often found to be relatively simple and quite similar. For example the Lorenz type behaviour in Fig. 3 is for a 3-level laser which has no clear mathematical equivalence to the 2-level Haken system. In other lasers for which there is adiabatic elimination of one or other of the fast variables, chaotic behaviour is precluded if, as a consequence, the number of variables of the system is reduced to less than three.

For these systems the addition of an independent external control parameter to the system, such as cavity length or loss modulation, have been extensively considered as a means to provide the extra degrees of freedom, as for the external drive of the Duffing oscillation considered above. In contrast for multimode rather than single-mode lasers, intrinsic modulation of inversion (or photon flux) by multimode parametric interaction ensures additional degrees of freedom. Furthermore, when the field is detuned from gain centre, the field amplitude, polarization and population inversion are complex, providing (in the absence of adiabatic elimination) five rather than three non-linear equations for single mode systems which is more than sufficient to yield deterministic chaos for suitable paramater values. Also of significance is the remarkably low threshold found for the generation of instabilities and chaos in single mode inhomogeneously broadened laser systems.
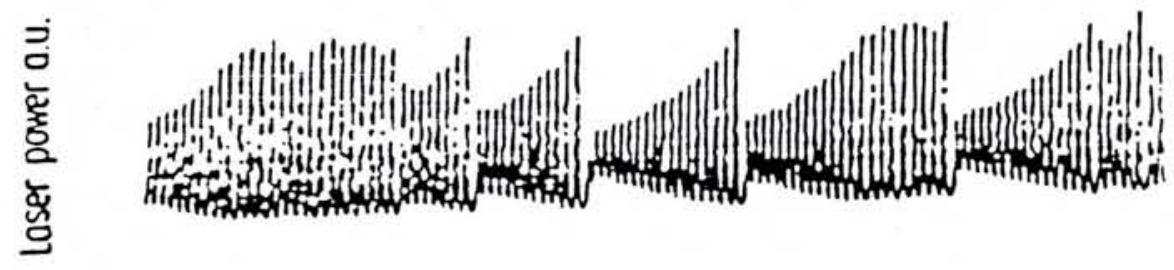

$$
\text { pulsing period - ips }
$$

Fig. 3 - Lorenz type chaos in $\mathrm{NH}_{3}$ laser emitting at $81 \mu$ m optically pumped by a $\mathrm{N}_{2} \mathrm{O}$ laser [9].

EPS Divisions, Sections and Group
Astronomy and Astrophysics Division
Solar Physics Section
Atomic and Molecular Physics Division
Atomic Spectroscopy Section
Chemical Physics
Electronic and Atomic Collisions
Molecular Physics
Computational Physics Group
Condensed Matter Division
Liquids Section
Low Temperature Physics Section
Macromolecular Physics
Magnetism
Metal Physics
Semiconductors and Insulators
Surfaces and Interfaces
High Energy \& Particle Physics Division
Interdiv. Group on Exptl. Phys. Control Systems
Interdiv. Group on Physics for Development
Nuclear Physics Division
Optics Division
Plasma Physics Division
Quantum Electronics Division
Europhysics News is the official joumal of the European
Physical Society which comprises 29 National Societies, Academies and Group, about 4000 Individual Members and 75 Associate Members. Governing bodies of EPS are the General Meeting, Council and an elected Executive Committee responsible for detailed policy. EPS promotes the collaboration of physicists throughout Europe, organising and harmonising conferences and publications, improving physics education, encouraging physics applications, awarding scholarships to sponsored schools in Erice. EPS publishes in addition to Europhys. News, Europhysics Letters lin partnership with national societies), European Journal of Physics (in partnership with The UK Inst. of Phys.), and European Conference Abstracts. Individual Members receive Europhys. News free of charge (price to insts.: Sw.Fr. 90.-la), Europhys. Lett. at Sw.Fr. 125./a (insts. 1050.-), rebates on many other publications and on conference fees. Annual EPS membership fee for Individual Members belonging to an EPS member society is: Sw.Fr. 44.-; independent members: Sw.Fr. 132.-members of a Collaborating Society: Sw.Fr. 55.- ciplinary field where in recent years there has been a proliferation of results from a wide range of laser systems.

\section{REFERENCES}

[1] Harrison R.G. and Biswas D.J., Prog. Quant. Elect. 10 (1985) 147.

[2] Thomson J.M.T. and Stewart H.B., Nonlinear Dynamics and Chaos (John Wiley, New York) 1987.

[3] Feigenbaum F.J., J. Stat. Phys. 19 (1978) 25.

[4] Manneville P. and Pomeau Y., Phys. Lett. 75A (1979) 1.

[5] Ruelle D. and Takens F., Commun. Math. Phys. 20 (1971) 167.

[6] Haken H., Phys. Lett. 53A (1975) 77.

[7]Lorenz E.N., J. Atoms Sci. 20 (1963) 130.

[8] Lanford O.E., Lecture Notes in Physics eds. P. Bernard and T. Raltu (Springer, Berlin) 1977.

[9] Weiss C.O. and Brocke J., Phys. Rev. Lett. 57 (1986) 2804. 